\title{
The Effects of Low Doses of Pregabalin on Morphine Analgesia in Advanced Cancer Patients
}

\author{
Sebastiano Mercadante, MD, ${ }^{*} \dagger$ Giampiero Porzio, MD, $\neq$ Federica Aielli, MD, $\ddagger$ \\ Patrizia Ferrera, MD,* Luigi Codipietro, MD,§ Claudio Lo Presti, MD,\| \\ and Alessandra Casuccio, BS
}

\begin{abstract}
Objectives: The aim of this study was to evaluate the opioid response in patients receiving morphine and pregabalin, independently from the presumed pain mechanisms, in comparison with patients receiving morphine treatment only.
\end{abstract}

Methods: A multicenter prospective randomized controlled study was carried out in a sample of 70 advanced cancer patients with pain requiring strong opioids. Thirty-five patients (group MO) were randomized to receive sustained-release morphine using initial doses of $60 \mathrm{mg} /$ day. Thirty-five patients (group MO-PR) were randomized to start the same morphine doses and pregabalin in increasing doses, starting with $25 \mathrm{mg} /$ day up to $150 \mathrm{mg} /$ day in one week. The following data were also recorded before starting the treatments (T0) and then at week intervals for four weeks (W1-4): age, gender, primary cancer and known metastases, pain causes and mechanisms, symptoms associated with opioid therapy, pain intensity, Brief Pain Inventory (BPI), morphine doses and escalation indexes (OEIs), and quality of life.

Results: Forty-eight patients completed the study, twenty-eight and sixteen patients in group MO and MO-PR, respectively. Twenty patients were females, the mean age was $65.5( \pm 10.3)$, and the mean Karnofsky status was $66.0( \pm 18.9)$. No differences between groups were found in age $(P=0.839)$, Karnofsky status $(P=0.741)$, opioid doses as well as escalation indexes (OEI mg, $P=0.260$, and OEI \%, $P=0.270$ ). No differences between the two groups were found in quality of life and all BPI items.

Conclusion: The use of low doses of pregabalin added to morphine therapy in advanced cancer patients does not seem to provide advantageous analgesic effects, despite limitations of the present study due to the number of drop-outs.

Key Words: cancer pain, neuropathic pain, assessment tools, opioid response

(Clin J Pain 2013;29:15-19)

ancer pain is a major clinical problem in patients with metastases. Like other chronic pain states, cancer pain involves sensitization of central neurons, associated with a

Received for publication January 4, 2011; revised September 30, 2011; accepted December 20, 2011.

From the *La Maddalena Cancer Center, Pain Relief and Palliative Care Unit; $†$ Department of Anesthesiology, Intensive Care and Emergency, and Palliative Medicine; 9 Department of Experimental Biomedicine and Clinical Neuroscience, University of Palermo; $₫$ Aquila per la vita, L’ Aquila; §Ospedale Parini, Aosta; and OOspedale San Filippo Neri, Rome, Italy.

The authors declare no conflict of interest.

Reprints: Sebastiano Mercadante, MD, La Maddalena Cancer Center, Pain Relief and Palliative Care Unit, Via San Lorenzo 312, 90146 Palermo, Italy (e-mails: terapiadeldolore@lamaddalenanet.it; 03sebelle@gmail.com).

Copyright (C) 2012 by Lippincott Williams \& Wilkins hyperactivity of $N$-methyl $D$-aspartate (NMDA)-type receptors. Other than oncological treatment for the primary disease, the current analgesic treatment includes specific measures, principally based on the guidelines provided by World Health Organization: a sequential approach of analgesics, step by step, according to the potency of drugs associated with adjuvants. Patients with moderate-to-severe pain are candidates to start strong opioids. ${ }^{1}$ Gabapentin has been used clinically for neuropathic cancer pain as an adjuvant agent. ${ }^{2-4}$ Recent reports have identified synergistic effects of gabapentic-like compounds, NMDA receptor antagonists, and opioids, because of the inhibition of afferent glutamate release. Different experimental models have shown the role of gabapentinoids in states of spinal cord hyperexcitation. NMDA hyperactivity is also involved in the development of tolerance and hyperalgesia to chronic treatment with opioids, ${ }^{5}$ states of spinal hyperexcitability in cancer-induced bone pain, ${ }^{6}$ and visceral hypersensitive states. ${ }^{7}$ A combination of morphine and gabapentin resulted in enhanced inhibitory effects on the evoked firing of dorsal horn neurons in a rat model of neuropathy. ${ }^{8}$

Experimental models have shown that bone pain, which is clinically associated with bone metastases and movementinduced pain, produce central sensitization leading to hyperalgesia, allodynia, and decreased response to opioids, in comparison with inflammatory models. ${ }^{9}$ In a case series, the addition of gabapentin in low doses was associated with significant improvement of incident pain exacerbated by movement, ${ }^{10}$ in patients with a presumably spinal hyperexcitatory state.

Pregabalin has a favorable pharmacokinetic profile compared with gabapentin. ${ }^{11,12}$ As with gabapentin, pregabalin is inactive at gamma-aminobutyric acid a and gamma-aminobutyric acid $b$ receptors, with the main site of action being the $\alpha 2-\delta$ subunit of presynaptic, voltagedependent calcium channels. As upregulation of the $\alpha 2-\delta$ subunit may play an important role in hypersensitization processes, pregabablin appears to play a potential role in inhibiting the modulation of neuronal excitability. ${ }^{13}$ Pregabalin dosing aimed at the optimal balance of efficacy and tolerability provided significant pain relief and reduced the risk of adverse effects and therapy discontinuation. ${ }^{14}$ The potential usefulness of calcium-channel ligands in combination with opioids is suggested by data from research and clinical trials. ${ }^{15}$

According to these observations the association of opioid therapy and pregabalin could be helpful in improving opioid analgesia, also braking the tendency to escalate the opioid doses in a long-term period, which could result in an increase of opioid-related adverse effects, other than the development of opioid-induced hyperalgesia. 
Relatively low doses of adjuvants may be better tolerated providing an opioid-sparing analgesia, according to their putative antihyperalgesic effects. ${ }^{16}$

The aim of this study was to evaluate the opioid response in patients receiving morphine and pregabalin, independently from the presumed pain mechanisms, in comparison with patients receiving morphine treatment only.

\section{PATIENTS AND METHODS}

A multicenter prospective randomized controlled study was carried out in a sample of 70 advanced cancer patients with pain requiring strong opioids, who had received opioids for mild-to-moderate pain, including tramadol and codeine at doses of at least $300 \mathrm{mg}$ and $180 \mathrm{mg}$, respectively, unsuccessfully. Patients with relevant coexisting liver or renal disease, cognitive impairment, an expected survival $<3$ months, requiring radiotherapy, or a new course of chemotherapy, with a prevalent incident pain component were excluded. The study was approved by the Local Ethical Committee of the University of Palermo, and adhered to Helsinki declaration (Eudract number 2008-005954-19).

Randomization was computer-generated. Thirty-five patients (group MO) were randomized to receive sustainedrelease morphine using initial doses of $60 \mathrm{mg} / \mathrm{d}$. Thirty-five patients (group MO-PR) were randomized to start the same morphine doses and pregabalin in increasing doses, starting with $25 \mathrm{mg} / \mathrm{d}$ up to $150 \mathrm{mg} / \mathrm{d}$ in 1 week. Oral morphine was provided in doses of about $1 / 6$ of their 24 -hour oral morphine equivalent requirement, as breakthrough pain medication. Doses were computed in the following days if $>2$ extradoses were required, according to department policy. ${ }^{17}$ The subsequent dosages of oral morphine were changed to obtain an adequate balance between acceptable analgesia and tolerable adverse effects. The doses of pregabalin were also increased according to the protocol, but a flexible use ${ }^{14,18}$ was allowed to find "the best tolerated dose" within a week. The use of other drugs was allowed, including ones generally administered in palliative care to manage symptoms. Patients already receiving nonopioid analgesics or steroids could continue their treatment, while all drugs with a putative analgesic effect, coanalgesics such as antidepressants or other anticonvulsants, were proscribed. All patients were strictly monitored by frequent phone contacts and regular visits. Patients with a poor opioid response, because of the prevalence of adverse effects, despite symptomatic treatment, were switched to an alternative opioid, and were withdrawn from the study.

The following data were also recorded before starting the treatments (T0) and then at weekly intervals for 4 weeks (W1 to W4). An extension period was also planned with a further evaluation after 8 weeks (W8), whenever possible:

- Age, sex, primary cancer and known metastases, pain causes and mechanisms on the basis of clinical history, known metastases, physical examination, and available investigations.

- Symptoms associated with opioid therapy, such as nausea and vomiting, drowsiness, and confusion, were recorded (assessed by patients), using a scale from 0 to 3 (not at all, slight, a lot, severe), unless for constipation, which was rated by the following scale used, according to monitoring policy at our institution: $0=1$ passage/ 1 to 2 days, $1=1$ passage $/ 3$ to 4 days, $2=1$ passage $>4$ days, $3=$ rectal measures. ${ }^{16}$

- Pain intensity, measured using the patient's self report on a numerical 0 to 10 scale.
- A Brief Pain Inventory (BPI) at the different intervals.

- From the opioid starting dose (OSD), $60 \mathrm{mg} / \mathrm{d}$ of morphine at referral, and the doses administered 4 weeks after (opioid maximum dose, the OEI as a percentage (OEI\%) was calculated according to the following formula: [(opioid maximum dose-OSD)/OSD]/days $\times 100 .{ }^{19}$

- At inclusion, on the basis of the patient's history, clinical examination performed by an expert physician with experience in neurological examination in cancer patients, and results from recent neuroimaging examinations, were collected. Patients were classified into tertiles based on ascending ratings of diagnostic certainty by clinicians, with graded evidence of nervous system lesion, and labeled as definite neuropathic pain (NP), possible $\mathrm{NP}$, or unlikely $\mathrm{NP},{ }^{16,20}$ according to the criteria for clinical classification of suspected NP suggested by Rasmussen et $\mathrm{al}^{21}$ and instrumental findings.

- Doses of pregabalin tolerated at the different time intervals chosen.

- Quality of life was evaluated by the Spitzer QoL Index, which is a cancer-specific measurement. The score was calculated after answering the 5 items, rated on a Likert 3 -point scale ( 0 to 2 ) in the areas of activity, daily life, health perceptions, social support, and behavior. ${ }^{22}$

\section{Statistical Analysis}

A power analysis indicates that a sample size of 25 patients per group would allow the detection of a $20 \%$ difference $(P<0.05$, power $=0.8)$. This computation assumes that the mean difference is 0.20 with a $95 \%$ confidence interval of 0.07 to 0.33 and the common withingroup SD of 0.28 . Frequency analysis was performed with $\chi^{2}$ test. The univariate repeated measures analysis (analysis of variance) and the paired Wilcoxon signed-rank test were used to compare the means or the scores of parametric or nonparametric variables, respectively, at the different time intervals. The 1-way analysis of variance and Mann-Whitney $U$ statistic test were used to compare the different parametric or nonparametric variables. All $P$ values were 2-sided and $P$ values $<0.05$ were considered statistically significant.

\section{RESULTS}

Ten patients ( 1 and 9 patients in groups MO and MO$\mathrm{PR}$, respectively) did not start the study after randomization, because they withdrew their consent to participate. Of the remaining 60 patients, 48 patients completed the study, 30 and 18 patients in groups MO and MO-PR, respectively. Twelve patients did not complete the study because they were lost to follow-up. Of them, in group MO, 2 patients died, 1 patient was admitted for melena and then switched to other opioids, and 1 patient was switched to another opioid. In group MO-PR, 3 patients died, 2 patients discontinued pregabalin because of adverse effects (leg edema and drowsiness, respectively), and 1 patient droppedout for poor compliance.

Of the 48 patients, 28 and 16 patients in groups $\mathrm{MO}$ and MO-PR, respectively, were followed for the entire period of study (W4), and 22 patients and 14 patients, respectively, for the extension up to W8. Twenty patients were female, the mean age was $65.5( \pm 10.3)$ years, and the mean Karnofsky status was $66.0( \pm 18.9)$. No differences between groups were found in age $(P=0.839)$, Karnofsky status $(P=0.741)$, 


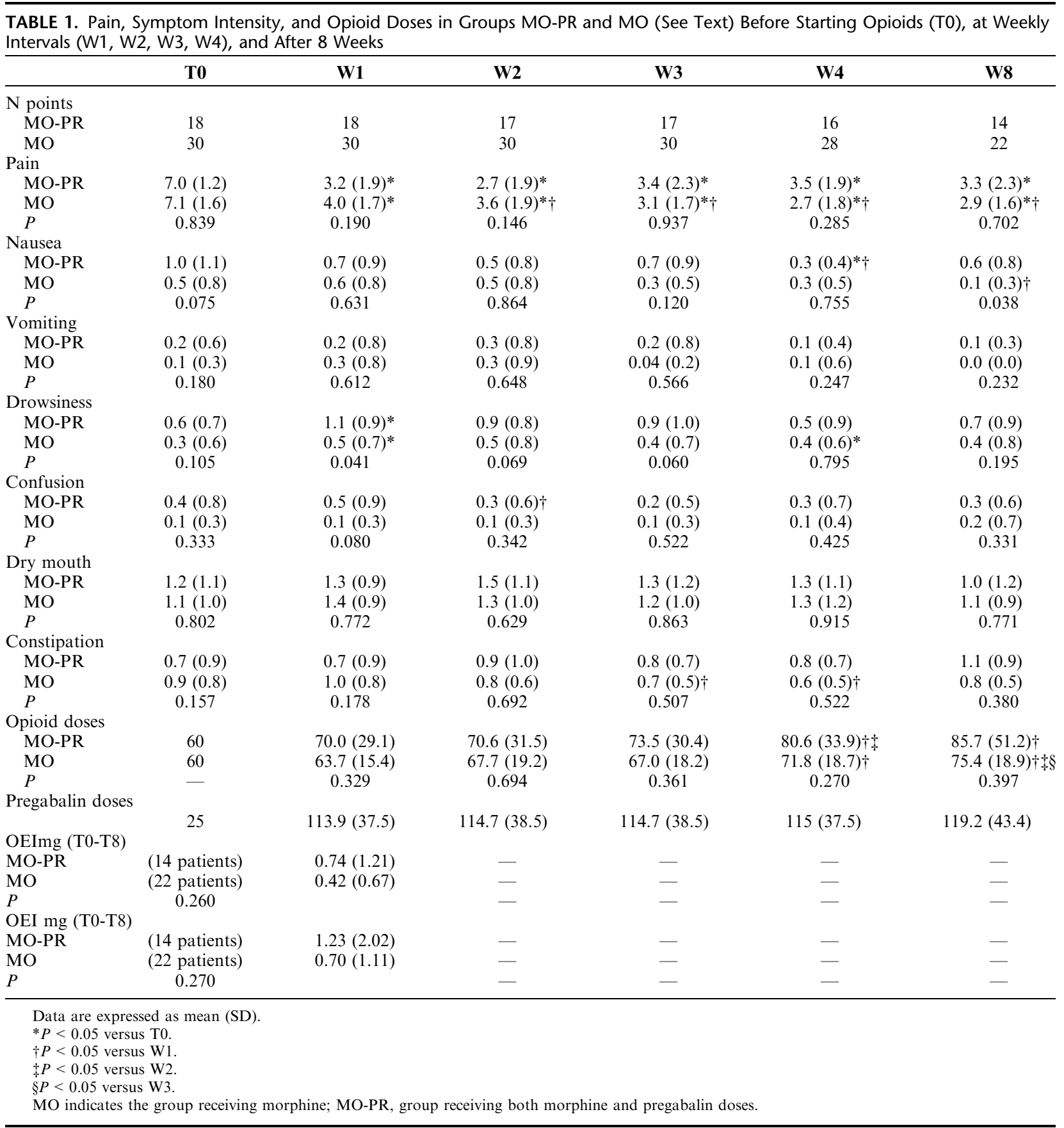

opioid doses, and the calculated escalation indexes (OEImg, $P=0.260$, and $\mathrm{OEI} \%, P=0.270$ ). Eight and 2 patients in groups MO and MO-PR, respectively, had "definite" neuropathic pain. This low number of patients did not allow a further subgroup analysis.

Data on pain and symptom intensity, morphine and pregabalin doses, and OEI are reported in Table 1. No statistical differences were observed. No differences between the 2 groups were found in quality of life and all BPI items (Table 2). In a subgroup analysis, 10 patients ( 8 and 2 patients in groups MO and MO-PR, respectively) had a definite neuropathic pain. As expected, this did not indicate a significant difference (Pearson $\left.\chi^{2}, 4.551\right)$. No differences in the number of patients receiving adjuvant or symptomatic drugs were found between the 2 groups.

\section{DISCUSSION}

Pregabalin plays a potential role in reducing pain, providing better analgesia in association with morphine in the management of cancer pain in advanced cancer patients. Gabapentinoids selectively reduced C-fiber-evoked field potentials after induction of long-term potentiation, suggesting that a combination of lower doses of gabapentinoids 
TABLE 2. Data on Quality of Life and BPI in the 2 Groups (MO-PR and MO) Before Starting Morphine (T0), at Weekly Intervals (W1, W3, W4), and After 8 Weeks

\begin{tabular}{|c|c|c|c|c|c|c|}
\hline & T0 & W1 & W2 & W3 & W4 & W8 \\
\hline \multicolumn{7}{|l|}{$\mathrm{N}$ points } \\
\hline MO-PR & 18 & 18 & 17 & 17 & 16 & 14 \\
\hline MO & 30 & 30 & 30 & 30 & 28 & 22 \\
\hline \multicolumn{7}{|l|}{ QoL Spitz } \\
\hline MO-PR & $6.1(1.8)$ & $6.3(1.4)$ & $6.9(1.1)$ & $6.7(2.0)$ & $6.6(2.1)$ & $7.1(1.5)$ \\
\hline MO & $5.4(2.0)$ & $6.2(1.3)^{*}$ & $6.6(1.5)^{* \dagger}$ & $6.7(1.6)^{*}$ & $6.7(1.8)^{*}$ & $7.0(1.1)^{*}$ \\
\hline$P$ & 0.333 & 0.775 & 0.826 & 0.865 & 0.920 & 0.756 \\
\hline \multicolumn{7}{|c|}{ BPI General activity } \\
\hline MO-PR & $6.7(2.7)$ & $4.2(2.5)^{*}$ & $4.3(2.5)^{*}$ & $4.5(3.0)^{*}$ & $4.2(2.0)^{*}$ & $2.9(2.4)^{*}$ \\
\hline MO & $6.8(1.8)$ & $4.9(1.9)^{*}$ & $4.5(1.9)^{*}$ & $4.1(1.8) * \dagger$ & $3.5(1.8)^{*}+\hbar$ & $3.7(1.7)^{*} \dagger$ \\
\hline$P$ & 0.866 & 0.369 & 0.603 & 0.726 & 0.340 & 0.199 \\
\hline \multicolumn{7}{|l|}{ BPI Mood } \\
\hline MO-PR & $6.7(2.3)$ & $5.1(2.5)^{*}$ & $3.8(2.9)^{*}$ & $3.8(3.3)^{*}$ & $3.5(2.5)^{*}$ & $2.5(2.7)^{*}$ \\
\hline MO & $6.6(2.1)$ & $4.4(1.9)^{*}$ & $4.3(2.0)^{*}$ & $3.9(1.9)^{* \dagger}$ & $3.4(1.7)^{*}+t$ & $3.3(1.5)^{*} \dagger \dagger$ \\
\hline$P$ & 0.728 & 0.230 & 0.450 & 0.589 & 0.988 & 0.314 \\
\hline \multicolumn{7}{|c|}{ BPI Walking } \\
\hline MO-PR & $5.2(3.6)$ & $4.3(3.5)$ & $3.5(3.6)$ & $3.7(3.2)$ & $3.8(2.8)$ & $3.2(3.1)$ \\
\hline MO & $6.0(2.4)$ & $4.1(1.9)^{*}$ & $3.8(1.9)^{*}$ & $3.6(2.1)^{*} \dagger$ & $2.8(1.5) * \dagger+\S$ & $2.9(1.4)^{*} \dagger \dagger$ \\
\hline$P$ & 0.391 & 0.929 & 0.253 & 0.772 & 0.420 & 0.662 \\
\hline \multicolumn{7}{|c|}{ BPI Working activity } \\
\hline MO-PR & $6.7(3.0)$ & $4.7(3.4)^{*}$ & $3.5(3.0)^{*}$ & $3.7(3.5)^{*}$ & $4.3(3.2)^{*}$ & $3.2(2.6)^{*}$ \\
\hline MO & $6.6(2.4)$ & $4.7(2.3)^{*}$ & $4.1(2.1)^{*}$ & $4.1(2.4)^{*}$ & $3.2(1.7)^{* \dagger}$ & $3.4(1.7)^{* \dagger} \dagger$ \\
\hline $\mathrm{P}$ & 0.760 & 0.886 & 0.421 & 0.453 & 0.449 & 0.520 \\
\hline \multicolumn{7}{|c|}{ BPI Relationship with other persons } \\
\hline MO-PR & $5.1(3.4)$ & $3.7(3.1)^{*}$ & $3.1(3.0)^{*}$ & $2.7(3.3)^{*}$ & $3.1(2.9)^{*}$ & $1.9(2.6)^{*}$ \\
\hline MO & $5.0(2.5)$ & $3.3(2.3)^{*}$ & $2.8(1.9)^{*}$ & $3.1(2.6)^{*}$ & $1.9(1.6)^{*}+\hbar \S$ & $2.0(1.4)^{*} \dagger t$ \\
\hline $\mathrm{P}$ & 0.667 & 0.528 & 0.826 & 0.372 & 0.371 & 0.349 \\
\hline \multicolumn{7}{|l|}{ BPI Sleep } \\
\hline MO-PR & $6.0(2.9)$ & $4.3(3.7)^{*}$ & $2.4(2.7)^{* \dagger}$ & $2.7(3.0)^{*}$ & $3.1(3.0)^{*}$ & $3.4(3.1)^{*}$ \\
\hline MO & $5.2(2.4)$ & $2.7(2.2)^{*}$ & $2.6(1.9)^{*}$ & $2.4(1.9)^{*}$ & $1.6(1.5)^{*+\dagger \S}$ & $1.7(1.5)^{*} \dagger+\delta$ \\
\hline $\mathrm{P}$ & 0.380 & 0.215 & 0.445 & 0.939 & 0.208 & 0.163 \\
\hline \multicolumn{7}{|c|}{ BPI Pleasure to live } \\
\hline MO-PR & $6.4(3.0)$ & $5.2(3.1)^{*}$ & $3.2(3.1)^{* \dagger}$ & $3.5(2.9)^{* \dagger}$ & $3.6(2.5)^{* \dagger} \dagger$ & $3.1(2.2)^{* \dagger} \dagger$ \\
\hline MO & $5.4(2.5)$ & $3.9(2.1)^{*}$ & $3.9(1.9)^{*}$ & $3.8(1.9)^{*}$ & $3.2(1.9)^{*}+\$ \S$ & $2.9(1.7)^{*} \dagger \pm \S$ \\
\hline $\mathrm{P}$ & 0.116 & 0.089 & 0.314 & 0.562 & 0.485 & 1.0 \\
\hline
\end{tabular}

Data are expressed as mean (SD).

$* P<0.05$ versus T0.

$\dagger P<0.05$ versus $\mathrm{W} 1$.

$\$ P<0.05$ versus $\mathrm{W} 2$.

$\S P<0.05$ versus W3.

BPI indicates Brief Pain Inventory; MO, group receiving morphine; MO-PR, group receiving both morphine and pregabalin doses.

and morphine produce better analgesic effects in chronic pain. ${ }^{23}$ In volunteers, gabapentinoids have been shown to enhance morphine analgesia. ${ }^{24}$ Pregabalin and morphine significantly reduced the area of secondary hyperalgesia. ${ }^{25}$ Moreover, gabapentinoids reverse microglial activation in the spinal cord. ${ }^{26}$ Pregabalin may also provide benefits to morphine analgesia without the danger of enhanced dependence liability. ${ }^{27}$

The present study was the first presenting data regarding the use of pregabalin added to morphine in advanced cancer pain. It was preferred to use a flexible approach in dosing pregabalin, avoiding a rigid protocol, to better reproduce the clinical setting and to extrapolate this information in clinical practice. It has been reported that the important methodological features that appeared to be feasible to reproduce data gathered from clinical trials in clinical practice were slow titration and flexible dosing. ${ }^{28}$

Data from the present study, however, did not confirm that pregabalin in doses of about $100 \mathrm{mg} / \mathrm{d}$ offers specific analgesic benefits to patient receiving morphine for chronic cancer pain. No differences in the decrease in pain intensity after starting similar doses of morphine were observed in patients who received pregabalin added to morphine therapy. In addition, despite a "flexible use" of pregabalin with a chance to change doses to limit the occurrence of adverse effects, according to recent suggestions, ${ }^{28}$ drowsiness was more intense in patients receiving pregabalin after 1 week of treatment. This effect, however, tended to disappear with the continuation of the treatment. In addition, the intensity of nausea was higher in patients receiving pregabalin at $\mathrm{W} 8$, although this late effect could be attributed to other concomitant causes.

Of interest, opioid doses did not differ between groups and OEIs used to monitor the dose escalation of morphine in time. Finally, no differences in BPI and quality of life were found, despite a positive trend in both groups in general.

Data from this study cannot exclude the fact that pregabalin would be more effective under more specific conditions such as neuropathic pain. Previous controlled studies of the parent drug have shown some efficacy of gabapentin in neuropathic cancer pain, ${ }^{2,4}$ and it is likely that pregabalin may reproduce similar effects. Unfortunately, a subgroup analysis of patients with "definite" neuropathic 
pain was unreliable for the lower number of patients, also because of the large number of dropouts. Thus, at the moment, no information exists supporting this hypothesis. Similarly, a possible effect on pain associated with bone metastases cannot be excluded. Alternately, higher doses could possibly be more effective, although this approach may produce more adverse effects.

This study has considerable limitations and this information should be taken cautiously. The principal weaknesses of this study include the relatively small size of the sample. The sample power dropped to $60 \%$ at the end of study, limiting the statistical validity. Moreover, the dropout rate was not equally distributed in the 2 groups, despite an appropriate randomization. Studies with drugs used for relatively prolonged periods of time are very difficult to perform, particularly if patients are then followed up by phone interviews and visits at the outpatient clinic. The absence of some differences does not constitute a claim for equivalence between the treatments, because of the inevitable number of dropouts. However, the dropout rate reported in this study was consistent with that observed in longitudinal studies of advanced cancer patients and reflects the difficulties in performing controlled trials in this population. ${ }^{29}$ Finally, the choice of not blinding the study was directed by the need to reproduce a daily clinical scenario allowing the therapeutic flexibility needed to reproduce what happens in the daily activity and to avoid any influence from any economical support from pharmaceutical industries.

In conclusion, the use of low doses of pregabalin added to morphine therapy in advanced cancer patients does not seem to provide advantageous analgesic effects. Further studies with appropriate design should explore whether pregabalin could exert an analgesic effect in patients with specific pain conditions, such as neuropathic pain or incident bone pain, or with a different dosing protocol.

\section{REFERENCES}

1. Hanks GW, De Conno F, Cherny N, et al. Morphine and alternative opioids in cancer pain: the EAPC recommendations. Br J Cancer. 2001;84:587-593.

2. Caraceni A, Zecca E, Bonezzi C, et al. Gabapentin for neuropathic cancer pain: a randomized controlled trial from the gabapentin cancer pain study group. $J$ Clin Oncol. 2004;22:2909-2917.

3. Ross JR, Goller K, Hardy J, et al. Gabapentin is effective in the treatment of cancer-related neuropathic pain: a prospective, open-label study. J Palliat Med. 2005;8:1118-1126.

4. Keskinbora K, Pekel AF, Aydinli I. Gabapentin and an opioid combination versus opioid alone for the management of neuropathic cancer pain: a randomized open trial. J Pain Symptom Manage. 2007;34:183-189.

5. Van Elstraete A, Sitbon P, Mazoit J, et al. Gabapentin prevents delayed and long-lasting hyperalgesia induced by fentanyl in rats. Anesthesiology. 2008;108:484-494.

6. Donovan-Rodriguez T, Dickenson AH, Urch K. Gabapentin normalizes spinal neuronal responses that correlate with behavior in a rat model of cancer-induced bone pain. Anesthesiology. 2005;102:132-140.

7. Houghton LA, Fell C, Whorwell PJ, et al. Effect of a secondgeneration $\alpha 2-\delta$ ligand (pregabalin) on visceral sensation in hypersensitive patients with irritable bowel syndrome. Gut. 2007;56:1218-1225.
8. Matthews EZ, Dickenson AH. A combination of gabapentin and morphine mediates enhanced inhibitory effects on dorsal horn, neuronal responses in a rat model of neuropathy. Anesthesiology. 2002;96:633-640.

9. Urch C. The pathophysiology of cancer-induced bone pain: current understanding. Palliat Med. 2004;18:267-274.

10. Caraceni A, Zecca E, Martini C, et al. Gabapentin for breakthrough pain due to bone metastases. Palliat Med. 2008;22:392-393.

11. Sills GJ. The mechanisms of action of gabapentin and pregabalin. Curr Opin Pharmacol. 2006;6:108-113.

12. Gajraj NM. Pregabalin: its pharmacology and use in pain management. Anesth Analg. 2007;105:1805-1815.

13. Fink K, Dooley DJ, Meder WP, et al. Inhibition of neuronal $\mathrm{Ca} 2+$ influx by gabapentin and pregabalin in the human neocortex. Neuropharmacology. 2002;42:229-236.

14. Freynhagen R, Strojek K, Griesing T, et al. Efficacy of pregabalin in neuropathic pain evaluated in a 12-week, randomized, double-blind, multicentre, placebo-controlled trial of flexible- and fixed-dose regimens. Pain. 2005;111:254-263.

15. Gilron I, Bailey JM, Tu D, et al. Morphine, gabapentin, or their combination for neuropathic pain. $N$ Engl $\mathrm{J} \mathrm{Med}$. 2005;352:1324-1334.

16. Bennett MI. Effectiveness of antiepileptic or antidepressant drugs when added to opioids for cancer pain: systematic review. Palliat Med. 2011;25:553-559.

17. Mercadante S, Ferrera P, Villari P, et al. Rapid switching between transdermal fentanyl and methadone in cancer patients. J Clin Oncol. 2005;23:5229-5234.

18. Vranken JH, Dijkgraaf MG, Kruis MR, et al. Pregabalin in patients with central neuropathic pain: a randomized, doubleblind, placebo-controlled trial of a flexible-dose regimen. Pain. 2008:136:150-157.

19. Mercadante S, Fulfaro F, Casuccio A, et al. Investigation of an opioid response categorization in advanced cancer patients. J Pain Symptom Manage. 1999;18:347-352.

20. Mercadante S, Bruera E. Opioid switching: a systematic and critical review. Cancer Treat Rev. 2006;32:304- 315.

21. Rasmussen PV, Sindrup SH, Jensen TS, et al. Symptoms and signs in patients with suspected neuropathic pain. Pain. 2004;110:461-469.

22. Sloan JA, Loprinzi CL, Kuross SA, et al. Randomized comparison of four tools measuring overall quality of life in patients with advanced cancer. J Clin Oncol. 1998;16:3662-3673.

23. Tanabe M, Murakami H, Honda $M$, et al. Gabapentin depresses $\mathrm{C}$-fiber-evoked field potentials in rat spinal dorsal horn only after induction of long-term potentiation. Exp Neurol. 2006;202:280-286.

24. Eckardt K, Ammon S, Hofmann U, et al. Gabapentin enhances the analgesic effect of morphine in healthy volunteers. Anesth Analg. 2000;91:185-191.

25. Wang H, Bolognese J, Calder N, et al. Effect of morphine and pregabalin compared with diphenhydramine hydrochloride and placebo on hyperalgesia and allodynia induced by intradermal capsaicin in healthy male subjects. J Pain. 2008; 9:1088-1095.

26. Wodarski R, Clark A, Grist J, et al. Gabapentin reverses microglial activation in the spinal cord of streptozocin-induced diabetic rats. Eur J Pain. 2009;13:807-811.

27. Andrews N, Loomis S, Blake R, et al. Effect of gabapentin-like compounds on development and maintenance of morphineinduced conditioned place preference. Psychopharmacology. 2001;157:381-387.

28. Katz N. Methodological issues in clinical trials of opioids for chronic pain. Neurology. 2005;65(suppl 4):S32-S49.

29. Mazzoccato C, Sweeney C, Bruera E. Clinical research in palliative care. J Palliat Med. 2001;115:261-264. 